\title{
Negative Filament Tension at High Excitability in a Model of Cardiac Tissue
}

\author{
Sergio Alonso ${ }^{1,2}$ and Alexander V. Panfilov ${ }^{3}$ \\ ${ }^{1}$ Abteilung Physikalische Chemie, Fritz-Haber-Institut der Max-Planck-Gesellschaft, Faradayweg 4-6, 14195 Berlin, Germany \\ ${ }^{2}$ Physikalisch-Technische Bundesanstalt, Abbestrasse 2-12, 10587 Berlin, Germany \\ ${ }^{3}$ Theoretical Biology, Utrecht University, Padualaan 83584 CH, Utrecht, The Netherlands
}

(Received 29 June 2007; published 28 May 2008)

\begin{abstract}
One of the fundamental mechanisms for the onset of turbulence in 3D excitable media is negative filament tension. Thus far, negative tension has always been obtained in media under low excitability. For this reason, its application to normal (nonischemic) cardiac tissue has been questionable, as such cardiac turbulence typically occurs at high excitability. Here, we report expansion of scroll rings (low curvature negative filament tension) in a medium with high excitability by numerical integration of the Luo-Rudy model of cardiac tissue. We discuss the relation between negative tension and the meandering of 2D spiral waves and the possible applications to cardiac modeling.
\end{abstract}

DOI: 10.1103/PhysRevLett.100.218101

PACS numbers: 87.19.Hh, 82.40.Bj, 87.19.R-

Rotating scroll waves have been found in various excitable media, such as the Belousov-Zhabotinsky reaction [1], aggregations of the slime mold Dictyostelium discoideum [2], and cardiac tissue [3], where they underlie life threatening cardiac arrhythmias [4]. Three-dimensional scroll waves rotate around regions of phase singularity, called filaments, and scroll wave dynamics are governed by the filament curvature, twist, and tension [5,6]. Typically, the filament has a positive linear tension; that is, it tends to reduce its length. As a result, scroll waves with a closed filament (scroll rings) shrink and disappear, whereas scroll waves with a nonclosed filament attain a stable shape [6]. However, it is also possible that filaments tend to increase their length, i.e., have negative filament tension. In this case scroll rings expand, which may result in an instability and onset of a turbulent excitation pattern [7-9]. Currently, the initial stage of negative filament tension has been observed in experiments with the Belousov-Zhabotinsky reaction [10]. It has been suggested that this instability can be responsible for the onset of turbulence in cardiac tissue resulting in ventricular fibrillation and sudden cardiac death $[7,11]$.

However, application of the idea of negative filament tension to cardiac arrhythmias is not straightforward. The main problem is that negative tension has always been observed in media with low excitability $[9,11,12]$, corresponding to parameter conditions near the failure of the wave propagation $[12,13]$. In such conditions the separation between the front and the back of the wave, called excitable gap, is substantial, the period of the scroll wave rotation is typically two or three times longer than the refractoriness of the medium, and the wave propagation velocity, even for solitary waves, is comparable with the failure value. On the contrary, cardiac tissue is highly excitable and it is well known that during ventricular fibrillation the excitable gap is very small, the rotation period of scroll waves is close to the refractory period of cardiac cells [14], and the velocity of the waves is substantially above the failure value [15].

In this Letter we report negative filament tension in a medium with high excitability. We show that for certain parameter values in an ionic model for cardiac tissue the filament of a scroll ring increases its length. We determine the parameter regions where this effect is observed and we study the relation between negative filament tension and the meandering patterns of 2D spiral waves. Although we did not observe breakdown to turbulence of wave patterns as a result of this instability, our study demonstrates that negative filament tension occurs in a parameter region with high excitability. This result may increase the importance of this mechanism for cardiology.

Model. - Propagation of the transmembrane potential in cardiac tissue is modeled by the following equation:

$$
\partial_{t} V=-I_{\text {ion }} / C_{m}+D \nabla^{2} V
$$

here, $V$ is the transmembrane potential, $t$ is time, $C_{m}=$ $1 \mu \mathrm{F} / \mathrm{cm}^{2}$ is the membrane capacitance, $D=$ $0.001 \mathrm{~cm}^{2} / \mathrm{ms}$ is the diffusion coefficient, and $I_{\text {ion }}$ is the sum of all transmembrane ionic currents. We use the LuoRudy phase 1 (LR1) model [16], which describes the biophysical mechanism of generation of action potential in cardiac cells by a relatively small number of state variables and was widely used to study wave propagation in 2D and 3D cardiac tissue. The representation of the total current is $I_{\text {ion }}=I_{\mathrm{Na}}+I_{\mathrm{si}}+I_{\mathrm{K}}+I_{\mathrm{K} 1}+I_{\mathrm{K} p}+I_{b}$, where $I_{\mathrm{Na}}=G_{\mathrm{Na}} m^{3} h j\left(V-E_{\mathrm{Na}}\right)$ is the fast $\mathrm{Na}^{+}$current, $I_{\mathrm{si}}=$ $G_{\mathrm{si}} d f\left(V-E_{\mathrm{si}}\right)$ is the slow inward $\mathrm{Ca}^{2+}$ current, $I_{\mathrm{K}}=$ $G_{\mathrm{K}} x x_{1}\left(V-E_{\mathrm{K}}\right)$ is the slow outward $\mathrm{K}^{+}$current, $I_{\mathrm{K} 1}=$ $G_{\mathrm{K} 1} \mathrm{~K} 1_{\infty}\left(V-E_{\mathrm{K} 1}\right)$ is the time-independent $\mathrm{K}^{+}$current, $I_{\mathrm{K} p}=G_{\mathrm{K} p} \mathrm{~K}_{p}\left(V-E_{\mathrm{K} p}\right)$ is the plateau $\mathrm{K}^{+}$current, and $I_{b}=G_{b}\left(V-E_{b}\right)$ is the background current [17]. For some simulations we clamped the gating variable $j$ to 1 , representing slow inactivation of $I_{\mathrm{Na}}$, to isolate the effect of spiral wave meandering [18]. Here we used the original 
values of the parameters given in [16], except for $G_{\mathrm{Na}}$ and $G_{\mathrm{si}}$, which were varied in our simulations (in [16] $G_{\mathrm{Na}}=$ $23 \mathrm{mS} / \mathrm{cm}^{2}$ and $G_{\mathrm{si}}=0.09 \mathrm{mS} / \mathrm{cm}^{2}$ ). Note that $G_{\mathrm{Na}}$ controls the speed of the waves [12] and in a general sense the excitability of the tissue, and $G_{\mathrm{si}}$ is responsible for the front-tail interaction between the pulses. Computations were performed using finite differences and an explicit time integration scheme on grids containing up to $200 \times$ $200 \times 200$ elements in $3 \mathrm{D}$ and $700 \times 700$ in $2 \mathrm{D}$, using Neumann boundary conditions and the integration steps $\Delta t=0.02 \mathrm{~ms}$ and $\Delta x=0.02 \mathrm{~cm}$. For further details see Ref. [12]. In addition to 2D and 3D simulations we also performed pseudo-3D simulations considering the cylindrical symmetry of scroll rings and reducing the 3D Laplacian operator to $\nabla^{2}=\partial_{r}^{2}+r^{-1} \partial_{r}+\partial_{z}^{2}[6,12]$.

Results. - We find that in some parametric region scroll rings can expand even if the medium is highly excitable (Fig. 1). We start with initial conditions in the form of a quarter of a scroll ring [Fig. 1(a)], which, due to the Neumann boundary conditions, is equivalent to a complete circular scroll ring. The time evolution of the filament is sketched in Fig. 1(b). We see that the scroll ring expands and shifts upwards, thus showing negative filament tension. The time course of the transmembrane potential is shown in Fig. 1(c). The excitable gap is small and the period of scroll wave rotation is close to the duration of the action potential.

Figure 2(a) shows the scroll ring radius from Fig. 1(a) in the course of time. We clearly see that after some transient the radius of the filament gradually increases, although the speed of expansion is low.

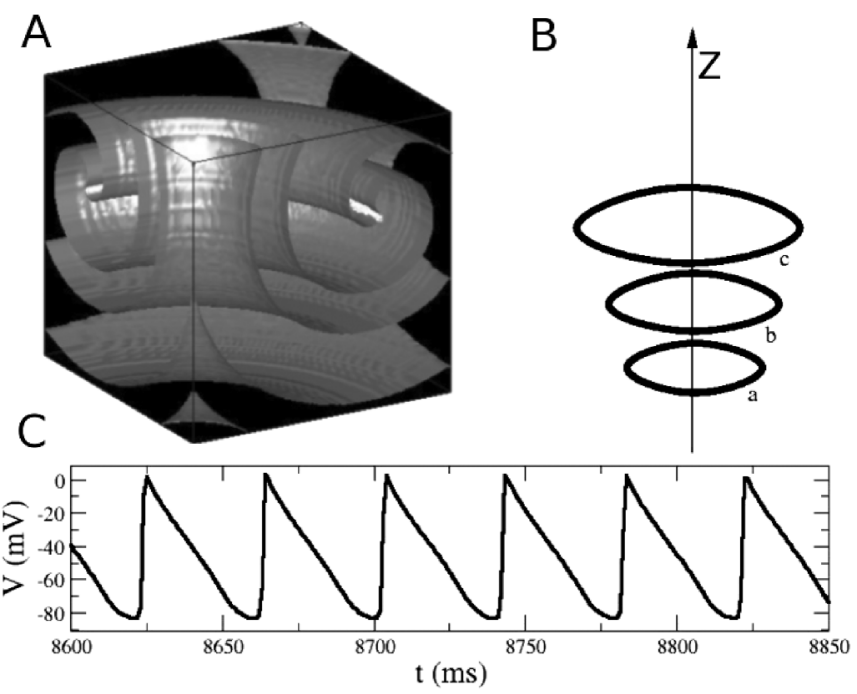

FIG. 1. Expansion of a quarter of scroll ring in the LR1 model at high excitability $\left(4 \times 4 \times 4 \mathrm{~cm}^{3}\right)$. (a) Wave pattern (gray) at $t=0$, the white line represents the filament. (b) Sketch of the successive filament positions $(a, b, c)$ in the course of time. (c) Transmembrane potential in the course of time at point $(0.2,0.2,0.04) . G_{\mathrm{Na}}=15.0 \mathrm{mS} / \mathrm{cm}^{2}$ and $G_{\mathrm{si}}=0.0 \mathrm{mS} / \mathrm{cm}^{2}$.
The expansion or collapse of scroll rings under high excitability depends on the initial radius. We see [in Fig. 2(b)] that if the initial radius is below $R_{c}=2.2 \mathrm{~cm}$, the scroll ring shrinks and collapses. For larger radius, scroll rings expand with a rate proportional to its curvature $\left(v_{r}=-\alpha / R\right)$ with $\alpha=-0.0003$ [19] for the parameter values used in Fig. 2(b). Such filament expansion for larger radius and collapse for small radius can be potentially understood from a stabilizing effect of the higher order terms in dependency of $v_{r}$ on curvature.

The velocity of expansion is low [note the difference of scale of $R$ between Figs. 2(a) and 2(b)], but it is robust and is not due to numerical or boundary effects (we obtained similar results for $\Delta x=0.00825 \mathrm{~cm}$ and $\Delta t=0.005 \mathrm{~ms}$ and for 3 times larger medium).

The overall diagram of filament dynamics in the parametric space $\left(G_{\mathrm{si}}, G_{\mathrm{Na}}\right)$ is shown in Fig. 3. Because negative filament tension in a medium with low excitability is closely related to the inward meandering of $2 \mathrm{D}$ spiral waves $[12,20,22]$ we also show here the type of the $2 \mathrm{D}$ meandering patterns. We identify two different regions of expansion of scroll rings, corresponding to the gray areas. The first is located in the limit of weak excitability, for $3.1<G_{\mathrm{Na}}<3.5 \mathrm{mS} / \mathrm{cm}^{2}$. This is the classical regime of scroll filament expansion in media with low excitability which we studied in [12]. In that case, scroll waves show a substantial excitable gap, and the region of negative filament tension is close to the nonexcitable zone and it corresponds to inward meandering or rigid rotation of spiral waves. A second region of scroll ring expansion is
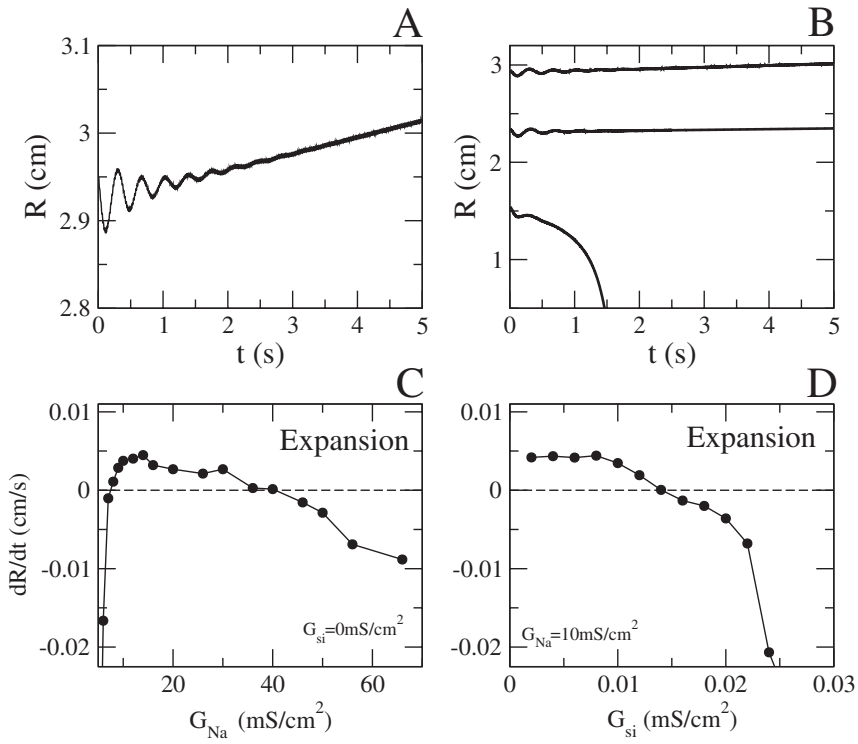

FIG. 2. (a) Radius of the scroll ring filament in the course of time for the 3D simulation from Fig. 1. (b) Evolution of the radius $(R)$ for different initial conditions. (c),(d) Dependence of the radial velocity on the parameters. Simulations in panels (c) and (d) are done with $2 \mathrm{D}$ cylindrical coordinates with $R_{0}=$ $3.5 \mathrm{~cm}$ and $j=1$. 


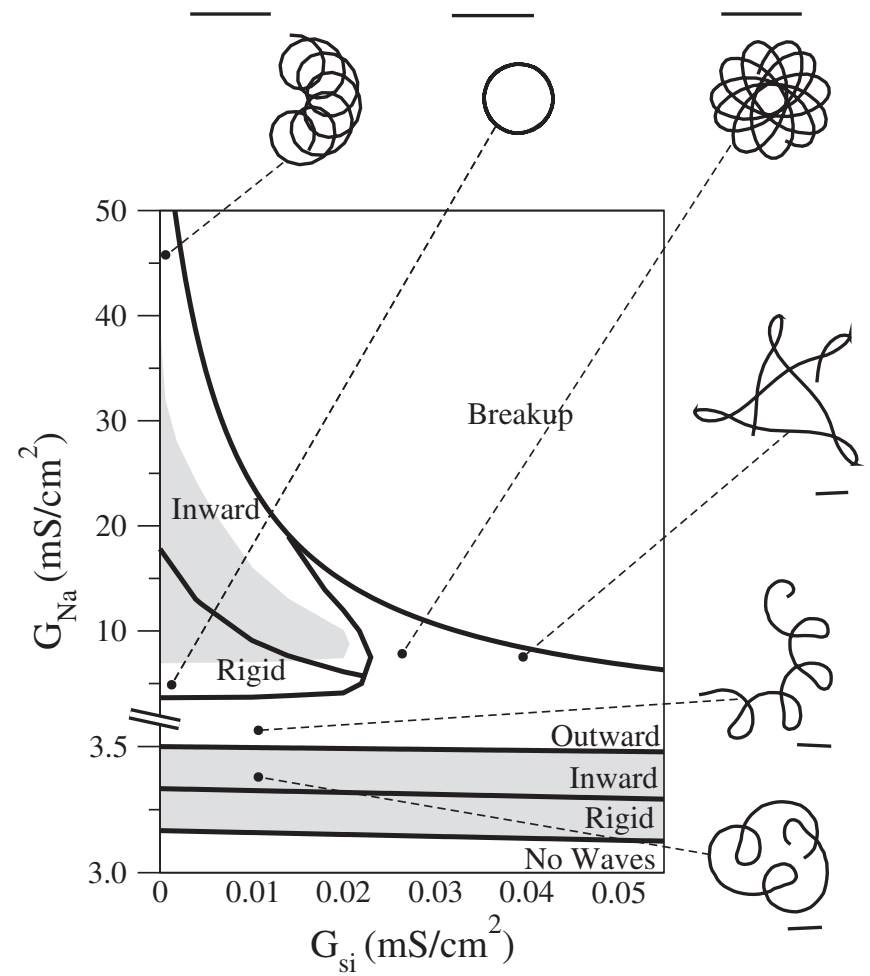

FIG. 3. Filament tension in $3 \mathrm{D}$ and $2 \mathrm{D}$ meandering patterns in parametric space for the LR1 model with $j$ clamped to 1 . The gray zones correspond to expansion of 3D scroll rings. Different regimes of spiral dynamics are indicated by the corresponding legends and are shown in the insets. The horizontal lines next to the meandering patterns are $2 \mathrm{~mm}$ long. 3D dynamics were computed for $R_{0}=3.5 \mathrm{~cm}$.

found in the medium with high excitability, and it is located at $8<G_{\mathrm{Na}}<35 \mathrm{mS} / \mathrm{cm}^{2}$. It is also related to a region of rigid rotation or inward meandering of $2 \mathrm{D}$ spiral waves, but in contrast to the low excitability case, the boundaries of negative filament tension do not precisely coincide with the boundaries of the different meandering regimes (see Fig. 3).

At low and high excitability there are two different types of inward meandering of spiral waves. Figure 4 shows the evolution of 2D meandering patterns under increasing $G_{\mathrm{Na}}$ and $G_{\mathrm{si}}$ for the cases of low and high excitability. Diagrams accompanying the tip paths in Fig. 4 show the structure of the meandering, typically a cycloid, with the direction of rotation of the tip around the core $\left(\omega_{1}\right)$ and the meandering rotation $\left(\omega_{2}\right)$. We see that in the case of low excitability the transition from inward to outward meandering occurs via linear drift of the spiral wave. The radius of the meandering increases and the frequency of the meandering decreases to zero as the parameter approaches the drift condition [23].

At high excitability the transition between inward and outward meandering is completely different. For low values of $G_{\mathrm{si}}$ the spiral waves perform an inward meandering, which is a cycloidal motion composed by two frequencies $\left(\omega_{1}, \omega_{2}\right)$. For a window of values of the parameter a new

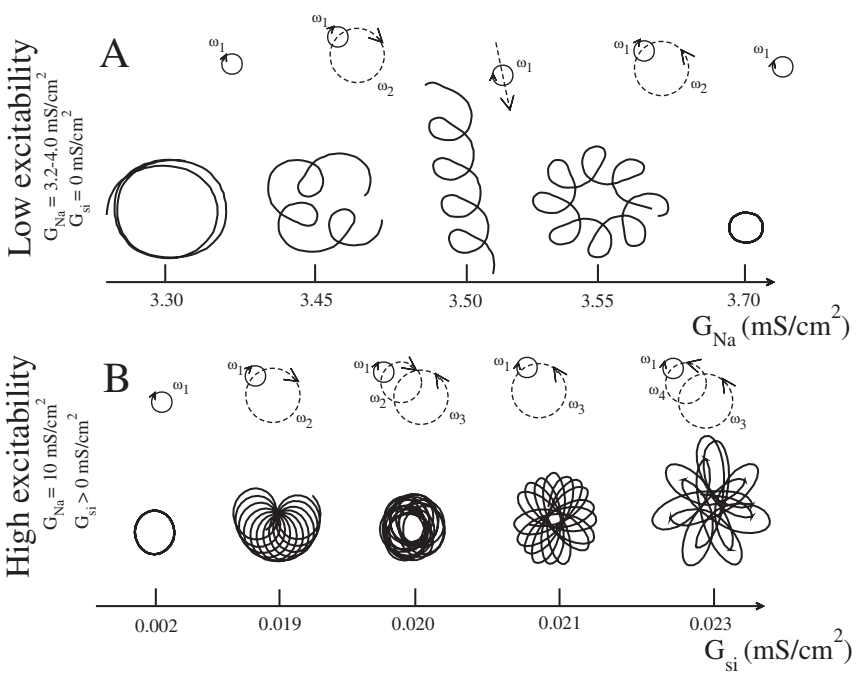

FIG. 4. Meandering of 2D spiral waves in the LR1 model. Transition between inward and outward meandering at low excitability for increasing $G_{\mathrm{Na}}$, at $G_{\mathrm{si}}=0 \mathrm{mS} / \mathrm{cm}^{2}$ (a) and under high excitability conditions for increasing $G_{\mathrm{si}}$ at $G_{\mathrm{Na}}=10 \mathrm{mS} / \mathrm{cm}^{2}$ (b). Gate variable $j$ is clamped to 1 .

third frequency $\left(\omega_{3}\right)$ appears, and the three frequencies are mixed in the motion of the spiral. Further increase of the parameter eliminates the original meandering frequency $\left(\omega_{2}\right)$ and the new one $\left(\omega_{3}\right)$ gives rise to outward meandering. For higher values of $G_{\mathrm{si}}$ the motion becomes more complex and new frequencies $\left(\omega_{4}\right)$ are needed to characterize the dynamics. Eventually, at $G_{\mathrm{si}}=0.03 \mathrm{mS} / \mathrm{cm}^{2}$ the spiral wave breaks down. The transition from negative to positive filament tension occurs around $G_{\mathrm{si}}=$ $0.015 \mathrm{mS} / \mathrm{cm}^{2}$, which is close to the region of onset of the third frequency in the meandering pattern in Fig. 4(b). In our case meandering with 3 or 4 frequencies is stable and spatially bounded and does not result in onset of chaotic behavior in cardiac tissue as, for example, during the process of spiral breakup, which may occur as a result of a quasiperiodic route to chaos [24].

We have also performed numerical simulations with the original LR1 model (without clamping of the gate variable

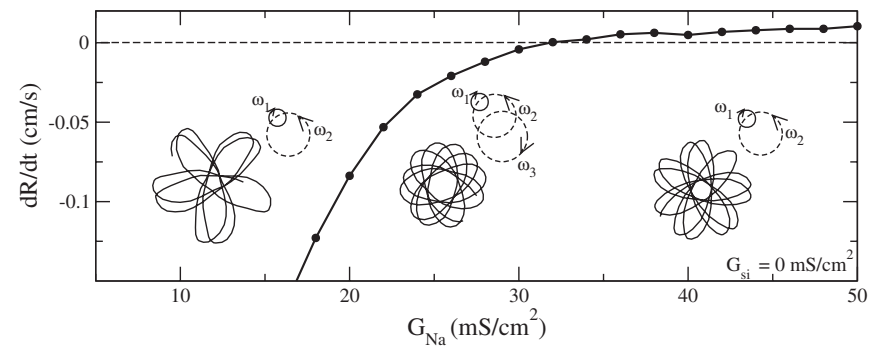

FIG. 5. Radial velocity of scroll rings for different values of the parameter $G_{\mathrm{Na}}$ at $G_{\mathrm{si}}=0 \mathrm{mS} / \mathrm{cm}^{2}$ for the full LR1 model (gate variable $j$ is unclamped). Such simulations have been done with cylindrical coordinates. Panels with trajectories of the spiral tip for several values of $G_{\mathrm{Na}}$ accompany the curve. 
$j$ ). We also found expansion of scroll rings at high excitability (Fig. 5). However, for the unclamped model there is no rigid rotation of spiral waves at high excitability [18], and the expansion of scroll rings in this case cannot be correlated with the rigid motion or inward meandering of the spiral wave. As we see in Fig. 5, scroll wave expansion occurs for $G_{\mathrm{Na}}>30 \mathrm{mS} / \mathrm{cm}^{2}$ for complex and outward meandering of spiral waves.

Discussion. - We show negative filament tension at low curvature in an ionic model of ventricular cardiac tissue under conditions of high excitability.

Typically, negative filament tension results in the onset of turbulent wave patterns. We do not find such turbulent regime in our simulations because the expansion of filament is small and therefore it could not induce cardiac fibrillation. However, this regime can still have important consequences for scroll wave dynamics in cardiac tissue. First, it will result in the absence of collapse of large $U$ shaped filaments, when they have small curvature. Second, instabilities induced by negative filament tension can be amplified by anisotropy or heterogeneity of cardiac tissue $[25,26]$.

Negative filament tension in low excitability is clearly related with the inward meandering of spiral waves $[11,12,20,22]$. Furthermore, as was shown in [21] the modes which determine meandering of spiral waves and the modes which determine the expansion of scroll rings are the same. We found that at high excitability the expansion of scroll rings in some cases can also be associated to the inward meandering of spiral waves, as shown in Fig. 3. For the full model (Fig. 5) we also observed a negative filament tension; however, its onset was not associated with inward meandering.

We think that in order to understand the mechanisms of negative filament tension at high excitability one needs to understand the origin of rigid rotation and inward meandering at high excitability. Although rigid rotation at high excitability looks similar to rigid rotation at the low excitable case, they have a different origin. In the low excitable situation the circular core is the result of curvature effects on spiral tip rotation [27] and is associated with a large excitable gap and absence of front-tail interactions. At high excitability, the circular core occurs with low excitable gap and strong front-tail interactions.

Note that inward meandering under high excitable conditions has previously been reported for other cardiac models [28] and in experiments on cardiac tissue [29]. Therefore, we think that similar studies performed in these systems might show negative filament tension.

In conclusion, we show negative filament tension at high excitability in an ionic model for cardiac tissue. Because $I_{\mathrm{Na}}$ descriptions are comparable in most ionic models, this study gives a guideline for finding this effect in other cardiac models. If the reactivation gating variable for the sodium current is clamped to a constant, the negative filament tension can be related to the rigid and inward meandering of the corresponding 2D spiral wave.

S. A. acknowledges financial support from the EU Training Network "Unifying principles in nonequilibrium pattern formation" and DFG within SFB 555. We thank K.H.W.J. ten Tusscher for providing software for this research and help in preparation of the manuscript.

[1] A. T. Winfree, Science 181, 937 (1973).

[2] F. Siegert and C. J. Weijer, Proc. Natl. Acad. Sci. U.S.A. 89, 6433 (1992).

[3] P. S. Chen et al., Circ. Res. 62, 1191 (1988); I. R. Efimov et al., J. Cardiovasc. Electrophysiol. 10, 1452 (1999).

[4] R. A. Gray and J. Jalife, Chaos 8, 65 (1998).

[5] J.P. Keener, Physica (Amsterdam) 31D, 269 (1988); S. Setayeshgar and A. J. Bernoff, Phys. Rev. Lett. 88, 028101 (2001).

[6] A. V. Panfilov and A. N. Rudenko, Physica (Amsterdam) 28D, 215 (1987).

[7] V. N. Biktashev, A. V. Holden, and H. Zhang, Phil. Trans. R. Soc. A 347, 611 (1994).

[8] S. Alonso et al., Science 299, 1722 (2003).

[9] S. Alonso et al., Phys. Rev. E 70, 056201 (2004).

[10] T. Bánsági et al., Phys. Rev. E 76, 045202(R) (2007); C. Luengviriya et al., Phys. Rev. Lett. 100, 148302 (2008).

[11] F. H. Fenton et al., Chaos 12, 852 (2002).

[12] S. Alonso and A. V. Panfilov, Chaos 17, 015102 (2007).

[13] V. Hakim and A. Karma, Phys. Rev. E 60, 5073 (1999).

[14] B. H. KenKnight et al., Circ. Res. 77, 849 (1995).

[15] H. J. Jongsma and R. Wilders, Circ. Res. 86, 1193 (2000).

[16] C. H. Luo and Y. Rudy, Circ. Res. 68, 1501 (1991).

[17] $E$ are the reversal potentials and $m, h, j, d, f$, and $x$ are gating variables, whose dynamics can be modeled by $\frac{d y}{d t}=$ $\left(y_{\infty}-y\right) / \tau_{y}$, where $y$ represents any of the gating variables, $y_{\infty}$ is the steady state value, and $\tau_{y}$ the relaxation time constant for the corresponding variable.

[18] Z. Qu et al., Ann. Biomed. Eng. 28, 755 (2000).

[19] This value was also confirmed using simulations of spiral drift in an external field and its relation to scroll ring curvature [20,21].

[20] H. Henry and V. Hakim, Phys. Rev. E 65, 046235 (2002).

[21] H. Henry, Phys. Rev. E 70, 026204 (2004).

[22] S. Alonso et al., J. Phys. Chem. A 110, 12063 (2006).

[23] D. Barkley, Phys. Rev. Lett. 72, 164 (1994).

[24] A. Garfinkel et al., J. Clin. Invest. 99, 305 (1997).

[25] F. H. Fenton and A. Karma, Chaos 8, 20 (1998).

[26] R. H. Clayton and A. V. Holden, Phys. Med. Biol. 48, 95 (2003).

[27] A.S. Mikhailov et al., Physica (Amsterdam) 70D, 1 (1994).

[28] J. Beaumont et al., Biophys. J. 75, 1 (1998).

[29] D. T. Kim et al., Chaos 8, 137 (1998). 